\title{
Requiem por la Atención de la Salud de la Mujer no Embarazada
}

\author{
Alberto Rizo Gil MD. MSP*
}

"Lo que más vale no es lo que se dice sino lo que se hace; son los actos de gobierno los que definen la política".

Hernán Durán $M$.

\section{ANTECEDENTES}

A partir de finales de 1987 el Ministerio de Salud Pública de Colombia introdujo modificaciones al Sistema de Programación de Servicios de Salud: se reajustó el modelo de programación para mejorar la atención de la demanda de la morbilidad y para reducir la mortalidad a niveles más bajos de los registrados en el año inmediatamente anterior.

Para atender los problemas de salud más agudos y buscarles soluciones adecuadas, se recomendó que la programación local de acciones de salud se orientara en forma especial a reducir las causas de muerte de aquellas enfermedades que con mayor frecuencia afectaban a la población.

Sin embargo al definir los grupos prioritarios y señalar las normas de atención para dichos grupos, los técnicos olvidaron la responsabilidad que tiene el Sector Oficial para programas intervenciones orientadas a mantener sanos a otros grupos específicos de la población, tales como el pre-escolar y la mujer en edad fértil, no embarazada.

Actividades de promoción de la salud destinadas a prevenir algunas enfermedades tales como la

Representante Regional para América Latina, the Pathfinder Fund. desnutrición proteico-calórica en el niño pre-escolar y otras como la planificación familiar cuya beneficiaria es la mujer sana no embarazada, fueron borradas de los formatos de programación.

Es muy posible sin embargo, que los organismos de salud hayan continuado atendiendo, como en el pasado, a pre-escolares y a mujeres que soliciten ser atendidas porque requieren información o servicios de anticoncepción. Sin embargo, será la demanda espontánea y no la búsqueda activa el factor que determine el número de consultas que se atienda -de tales grupos-por parte de los organismos del Sistema Nacional de Salud, como consecuencia de las modificaciones introducidas en la programación.

No es necesario mencionar el porqué gobiernos como los de Colombia sin distingos de color, principios, u orientación política, se han comprometido desde mediados de los años sesenta a incrementar el esfuerzo del sector salud para lograr la reducción de los riesgos de enfermar y morir en grupos tan vulnerables como el de la madre y el niño.

\section{EJEMPLO DE COMO PROGRAMAN ACCIONES MATERNO INFANTILES EN 1988. \\ EL CASO DE UN SERVICIO DE SALUD MODELO}

Me sirve para ilustrar lo que he afirmado sobre la "desaparición de las mujeres no embarazadas de la Programación Materno Infantil”, cifras reales del Servicio Seccional de Salud del Departamento del Quindío durante los años de 1987 y 1988, 13.

El Cuadro 1 permite conocer que para atender a 9.631 niños menores de 1 año y 9.977 mujeres embarazadas durante el año de 1988, el Servicio 
CUADRO 1

\section{SERVICIO SECCIONAL DE SALUD DEL QUINDIO PROGRAMACION DE ACTIVIDADES DE CONSULTA MEDICA Y DE ENFERMERIA EN EL AREA MATERNO INFANTIL}

\begin{tabular}{|c|c|c|c|c|c|}
\hline \multirow[t]{2}{*}{$\begin{array}{l}\text { Grupos } \\
\text { Atenc. }\end{array}$} & \multirow[t]{2}{*}{$\begin{array}{l}\text { Población } \\
\text { Asignada }\end{array}$} & \multicolumn{2}{|c|}{$\begin{array}{c}\text { Consultas o } \\
\text { Controles de Salud }\end{array}$} & \multicolumn{2}{|c|}{$\begin{array}{l}\text { Recursos disponib. } \\
\text { Para Atend. Cons. }\end{array}$} \\
\hline & & X Médico & $\begin{array}{l}\text { X Pers. Enf. } \\
\text { Enfermería }\end{array}$ & X Med. & $\begin{array}{l}\text { Por Pers. } \\
\text { Enfermer. }\end{array}$ \\
\hline $\begin{array}{l}\text { Niños } \\
\text { Men. } 1 \\
\text { Año }\end{array}$ & 9.631 & 28.593 & 36. 168 & 9.715 & 17.227 \\
\hline $\begin{array}{l}\text { Niños } \\
1-4\end{array}$ & * & & & & \\
\hline $\begin{array}{l}\text { Mujeres } \\
\text { No embaraz. }\end{array}$ & * & & & & \\
\hline $\begin{array}{l}\text { Mujeres } \\
\text { Embaraz. }\end{array}$ & 9.977 & 39.031 & 30.354 & 19.261 & 10.085 \\
\hline Totales & 19.608 & $\begin{array}{r}67.624 \\
134\end{array}$ & $\begin{array}{l}66.532 \\
4.156\end{array}$ & $\begin{array}{r}28.976 \\
56\end{array}$ & $\begin{array}{l}27.312 \\
288\end{array}$ \\
\hline
\end{tabular}

* Información no consignada por el Servicio Seccional de Salud.

cio Seccional de Salud del Quindío contaba con 56.288 horas médicas** y de enfermería o sea que cada persona en los grupos anotados, en promedio podía consultar 2.8 veces durante el año con base en los recursos existentes en dicho Servicio Seccional de Salud.

Como puede observarse, ni el niño en edad preescolar, ni la mujer no embarazada contaron con horas médicas o de personal de enfermería programadas por los Organismos del Servicio de Salud de ese departamento durante 1988. En la práctica, su atención se redujo a atender la demanda espontánea.

Veamos a continuación la situación existente en el mismo departamento durante el año de 1987, cuando la Programación de Servicios de Salud incluía al pre-escolar y a la mujer no embarazada como beneficiarios de acciones de salud para atender mejor la situación.

\footnotetext{
** 1 Médico trabajar do 8 horas diarias durante 250 días hábiles
} por un año totaliza 2.000 horas/año.
El Cuadro 2, nos permite apreciar que durante 1987, 323.189 personas en cuatro subgrupos específicos, cada uno de ellos con características bien estudiadas y conocidas, tuvieron asignadas 40.552 horas médicas y de personal de enfermería para ofrecerles un promedio de 1.25 consultas y controles al año por el Servicio de Salud. Puede observarse cómo al niño en edad pre-escolar se le asignó una cantidad importante de recursos médicos y de enfermería (un poco más de 20.000 horas totales al año de recurso médico y de enfermería). A las mujeres no embarazadas se les habían asignado 4.319 horas totales durante ese año, muy seguramente para atender la consulta de planificación familiar.

\section{CUADRO 2}
PROGRAMACION DE ACTIVIDADES MATERNO
INFANTILES POR EL SERVICIO SECCIONAL DE SALUD DEL QUINDIO DURANTE

EL AÑO DE 1987

(INCLUYE MEDICO Y PERSONAL DE ENFERMERIA)

\begin{tabular}{|c|c|c|c|c|c|}
\hline \multirow{3}{*}{$\begin{array}{l}\text { Grupos de } \\
\text { Atención }\end{array}$} & \multirow{3}{*}{$\begin{array}{l}\text { Población asig- } \\
\text { nada al servic. }\end{array}$} & \multicolumn{2}{|c|}{ Cons. o contr. } & \multirow{2}{*}{\multicolumn{2}{|c|}{$\begin{array}{l}\text { Recursos en } \\
\text { Disp. para } \\
\text { Atender las } \\
\text { Consultas }\end{array}$}} \\
\hline & & \multirow[t]{2}{*}{ X Med. } & \multirow{2}{*}{$\begin{array}{l}\text { X Pers. } \\
\text { Enferm. }\end{array}$} & & \\
\hline & & & & X Med. & $\begin{array}{l}\text { X Pers. } \\
\text { Enfer. }\end{array}$ \\
\hline \multicolumn{5}{|l|}{ Mende 1} & 5.453 \\
\hline \multicolumn{6}{|l|}{ Niños $1-4$} \\
\hline \multicolumn{3}{|l|}{ Pacientes } & 17.214 & 2.788 & 5.738 \\
\hline Usuarias de & 11.029 & 5.056 & 8.694 & 1.421 & 2.898 \\
\hline \multirow[t]{2}{*}{ Totales } & 32.198 & 46.380 & 99.220 & 12.930 & 27.622 \\
\hline & & \multicolumn{2}{|c|}{145.600} & \multicolumn{2}{|c|}{40.552} \\
\hline
\end{tabular}

La comparación de las tablas anteriores, si bien corresponde a los datos de un Servicio de Salud únicamente y no a la totalidad del país permiten obtener las siguientes conclusiones:

1a. A partir de 1988, los funcionarios que dictan las normas de programación en el Ministerio de Salud de Colombia decidieron excluir de la programación las actividades de fomento de la salud del niño en edad pre-escolar y a la mujer entre los 15 y 44 años, no embarazadas.

2a. Los grupos del niño menor de 1 año y la mujer embarazada están recibiendo atención prioritaria por parte del Sistema de Salud tan- 
to para incrementar su cobertura como para aumentar el volumen de actividades programadas a cada individuo en cada uno de estos dos grupos.

3a. Las horas de atención que prestaba el personal de enfermería durante 1987 (para los 4 grupos de riesgo pre-escolar y mujeres no embarazadas) se dedican a partir de 1988 en buena parte a atender sólo al menor de 1 año y a la embarazada.

4a. Se incrementa 2.25 veces los recursos médicos (horas médicas) entre 1987 y 1988 para atención del grupo del menor de un año y de la mujer embarazada.

5a. Presumiblemente en los organismos de salud oficiales se deja a la mujer no embarazada y al niño pre-escolar, algunas horas médicas y de enfermería para atender la demanda de morbilidad que presenten estos 2 grupos. Desaparecen la promoción y el fomento al menos en los formatos oficiales de programación.

\section{DISCUSION}

El ejemplo presentado sobre la manera como el Ministerio de Salud modificó la forma de programar acciones de salud para los grupos materno e infantiles en Colombia dejando de lado dos grupos importantes de población como son el pre-escolar y la mujer no embarazada, pone de manifiesto cómo las decisiones adoptadas por los niveles políticos se ignoran o no se interpretan en forma adecuada por los niveles técnicos (intermedios) en la organización rectora de la salud.

El examen de las actividadesque programan los Servicios de Salud orientadas desde las Oficinas de Planeación del Ministerio permite concluir que las acciones de fomento y prevención tienen poca importancia en los planes de salud que deben elaborar los Servicios Seccionales. Se podría afirmar que lo que interesa prioritariamente al Sistema de Salud hoy en día, son aquellas intervenciones que buscan reducir los daños que tienen los datos que ocupan los primeros lugares de morbilidad y la mortalidad.

Algunos técnicos afirman que Colombia no puede darse el lujo de utilizar recursos del Sistema de Salud en el fomento de la salud y la prevención de la enfermedad porque el país posee recursos limitados. Ejemplos como los de Chile y Costa Rica en este hemisferio constituyen una demostración sobre cómo aún con recursos limitados, la prevención y el fomento en esos países reciben atención adecuada.

Una administración responsable no puede ni debe permitirse descuidar tales acciones porque inciden para reducir la morbilidad y la mortalidad especialmente en grupos de riesgo elevado como los niños y las mujeres. Sería recomendable que las actuales autoridades de Planeación y de la División Materno Infantil del Ministerio de Salud Pública revisaran la decisión adoptada.

Documentos de programación producidos en el país hace 20 años cuando el entonces incipiente Programa de Salud Materno Infantil del Ministerio de Salud Pública de Colombia tomó la decisión de introducir por primera vez en la programación, acciones de salud en las áreas de fomento y prevención tales como el control y supervisión del crecimiento y desarrollo del niño, las visitas del post-parto, la planificación familiar, la detección precoz del cáncer ginecológico, etc. permiten entender cómo una decisión política era entonces respaldada plenamente por los niveles técnicos de la administración.

Las actividades mencionadas jamás hubieran sido programadas si para su jerarquización y ordenamiento se hubiera recurrido a los listados en donde se reportaban la morbilidad y las muertes.

Es indudable que el país ha retrocedido en este aspecto por cuanto no es lo mismo para un Sistema de Salud el esperar solamente a que la demanda se produzca. La búsqueda activa de grupos tales como el pre-escolar y la mujer no embarazada, es objeto de consideraciones especiales en todos los planes de salud de casi todos los gobiernos de este hemisferio. Aún no es muy tarde para revocar una decisión apresurada que está afectando a 2 grupos prioritarios de la población cuyo estado de salud debe mejorarse para que el país pueda alcanzar la "meta de salud para todos en el año 2.000" adoptada en Alma Ata.

\section{SUMMARY}

The Programming of Maternal and child health activities by the Ministry of Public health in Colombia was modified late in 1987. Pregnant women and infants were targeted as priority groups for health interventions in order to reduce morbidity and prevent deaths from occurring in both groups. 
The pre-school child and women in fertile age were left out from the programming as the resources used in previous years to take care of them were re-assigned to cover the infant and pregnant women only.
Health promotion and preventive intervention are essential and should not be discontinued by the Ministry of Health.

Data from Quindio, a central Department in Colombia, illustrates what has happened.

\section{BIBLIOGRAFIA}

1. Programación de Servicios de Salud. Módulo Autoinstrucción Ministerio de Salud Pública, octubre de 1987, Bogotá, Colombia.

2. Servicio Seccional de Salud del Quindío, Programación Materno Infantil, consulta general. Años 1987-1988.

3. Plan de Lucha contra la Pobreza Absoluta y para la generación de Empleo: Programa de Salud Básica para todos. Presidencia de la República, Bogotá, Colombia, 1986.
4. Módulo de Programación para el Sistema Nacional de Servicios de Salud. Chile, 1986 boletín Escuela de Medicina Universidad Católica de Chile. Santiago de Chile, Vol. 16 (2) 1986.

5. Programa de Extensión de Servicios de Protección Materna e Infantil y de Bienestar Familiar: Plan de Operaciones. Ministerio de Salud Pública, Bogotá, Colombia, marzo/69. 